\title{
Hybrid MR-PET of Brain Tumours using Amino Acid PET and Chemical Exchange Saturation Transfer MRI
}

\author{
N. A. da Silva ${ }^{a}$, P. Lohmann ${ }^{a}$, J. Fairney ${ }^{\text {b,c }}$, A. W. Magill ${ }^{a, d}$, A.-M. Oros Peusquens ${ }^{a}$, \\ C.-H. Choi ${ }^{\mathrm{a}}$, R. Stirnberg ${ }^{\mathrm{a}, \mathrm{e}}$, G. Stoffels ${ }^{\mathrm{a}}$, N. Galldiks ${ }^{\text {a, f, g }}$, X. Golay ${ }^{\text {b, c }}$, K.-J. Langen
} $\mathrm{a}, \mathrm{h}, \mathrm{i}$ and N. Jon Shah $\mathrm{a}, \mathrm{i}, \mathrm{j}$

${ }^{a}$ Institute of Neuroscience and Medicine, Forschungszentrum Jülich GmbH, Jülich, Germany

${ }^{\mathrm{b}}$ Department of Medical Physics \& Biomedical Engineering, University College London, London, London, United Kingdom

${ }^{\mathrm{c}}$ Department of Brain Repair and Rehabilitation, UCL Institute of Neurology, London, London, United Kingdom

${ }^{d}$ Current Address: Medical Physics in Radiology, German Cancer Research Centre (DKFZ), Heidelberg, Germany

${ }^{\mathrm{e}}$ German Center for Neurodegenerative Diseases (DZNE), Bonn, Germany

fDepartment of Neurology, University of Cologne, Cologne, Germany.

${ }^{g}$ Center of Integrated Oncology (CIO), Universities of Cologne and Bonn, Cologne, Germany

hJuelich-Aachen Research Alliance (JARA), Section JARA-Brain;

${ }^{\mathrm{i}}$ Department of Nuclear Medicine, RWTH University of Aachen, Aachen, Germany

${ }^{j}$ Department of Neurology, Faculty of Medicine, RWTH Aachen University, Aachen, Germany

*Corresponding Author:

Professor N. Jon Shah

Institute of Neuroscience and Medicine - 4

Forschungszentrum Jülich

52425 Jülich

Germany

Tel: +49 2461616836

Fax: +49 2461618294

Email:n.j.shah@fz-juelich.de 


\begin{abstract}
Purpose

PET using radiolabelled amino acids has become a promising tool in the diagnostics of gliomas and brain metastasis. Currently, amide proton transfer (APT) chemical exchange saturation transfer (CEST) MR imaging is evaluated for brain tumour imaging. In this hybrid MR-PET study, we compared in brain tumours with 3D data derived from APT-CEST MRI and amino acid PET using O- $\left(2-{ }^{18} \mathrm{~F}-\right.$ fluoroethyl)-L-tyrosine $\left({ }^{18} \mathrm{~F}-\mathrm{FET}\right)$.
\end{abstract}

\title{
Methods
}

Eight patients with gliomas were investigated simultaneously with ${ }^{18}$ F-FET PET and APT-CEST MRI using a 3 T MR-BrainPET scanner. CEST imaging was based on a steady-state approach using a $\mathrm{B}_{1}$ average power of $1 \mu \mathrm{T} . \mathrm{B}_{0}$ field inhomogeneities were corrected and parametric images of magnetisation transfer ratio asymmetry $\left(\mathrm{MTR}_{\text {asym }}\right)$ and differences to the extrapolated semi-solid magnetisation transfer reference method, APT\# and nuclear Overhauser effect (NOE\#), were calculated. Statistical analysis of the tumour-to-brain ratio of the CEST data was performed against PET data using the non-parametric Wilcoxon test.

\section{Results}

A tumour-to-brain ratio derived from APT\# and ${ }^{18} \mathrm{~F}$-FET presented no significant differences and no correlation was found between APT\# and ${ }^{18}$ F-FET PET data. Distance between local hot spots APT\# and ${ }^{18} \mathrm{~F}$-FET were different (average $20 \pm 13 \mathrm{~mm}$, range 4 - $45 \mathrm{~mm}$ ).

\section{Conclusion}

For the first time CEST images were compared with ${ }^{18} \mathrm{~F}$-FET in a simultaneous MR-PET measurement. Imaging findings derived from ${ }^{18} \mathrm{~F}$-FET PET and APT CEST MRI seems to provide different biological information. The validation of imaging findings by histological confirmation is necessary, ideally using stereotactic biopsy.

Keywords: CEST; APT; ${ }^{18}$ F-FET PET; hybrid MR-PET; glioma; 


\section{Introduction}

Diagnostics of brain tumours remains unsatisfactory despite high-resolution morphological imaging with conventional magnetic resonance imaging (MRI), in particular in pre-treated patients [1]. Multiple imaging modalities may be combined in order to improve diagnostic accuracy, such as advanced MRI and positron emission tomography (PET). In brain tumours, information about amino acid transport has become an important additional tool to standard MRI and has shown potential to define biological tumour volume for treatment planning, treatment monitoring and recurrence evaluation [2]-[8]. Several amino acid tracers are available, such as L-[methyl- $\left.{ }^{11} \mathrm{C}\right]-$ methionine $\left({ }^{11} \mathrm{C}-\right.$ MET), O- $\left(2-{ }^{18} \mathrm{~F}\right.$ - fluoroethyl)-L-tyrosine $\left({ }^{18} \mathrm{~F}-\mathrm{FET}\right)$ or 3,4 -dihydroxy- $6-\left[{ }^{18} \mathrm{~F}\right]$ fluoro-L-phenylalanine $\left({ }^{18} \mathrm{~F}-\mathrm{FDOPA}\right)$. Fluorine-18 labelled radiotracers are preferred due to the logistical advantages of the longer half-life. Furthermore, ${ }^{18} \mathrm{~F}$-FET exhibits different tracer kinetics depending on the tumour grade when compared to ${ }^{18} \mathrm{~F}$-FDOPA or ${ }^{11} \mathrm{C}-\mathrm{MET}[4]$.

Chemical exchange saturation transfer (CEST) is an MRI technique that enables the indirect detection of metabolites using the MR free water signal [10]. CEST is based on the phenomenon of transferring saturation from a solute pool (metabolites) to the water pool resulting in contrast that is dependent on metabolite concentration and its proton exchange rate [11], [12]. Repeating the measurements at different saturation frequencies allows the acquisition of the $\mathrm{Z}$-spectrum, where the contrast of multiple metabolites is encoded. This technique has many applications such as measurements of glycogen (GlycoCEST) [13], glutamate (GluCEST) [14], glucose (GlucoCEST) [15] and amide proton transfer (APT) [16]. Moreover, CEST contrast is also pH dependent [16]-[19]. APTCEST, which provides information at $3.5 \mathrm{ppm}$ downfield from water, has been shown to allow differentiation between low- and high-grade gliomas [20], [21]. In addition, APT-CEST has also shown its potential to distinguish between recurrent tumour and radiation necrosis [22], [23]. Furthermore, the signals from the upfield nuclear Overhauser effect $(\mathrm{NOE})$ at $-3.5 \mathrm{ppm}$ were also presented as a unique contrast in gliomas at $7 \mathrm{~T}$ [24]-[26].

The introduction of hybrid MR-PET scanners promotes the synergy between both modalities by allowing the simultaneous acquisition of human MRI and PET data [27]. However, to the best of our knowledge, no study in the literature has compared amino acid PET of brain tumours with APTCEST using simultaneous MR-PET. Initial attempts to compare CEST signals from amine and ${ }^{18} \mathrm{~F}$ FDOPA using non-simultaneous measurements have recently been reported [28], [29]. This is the first 
study to investigate simultaneous MR-PET brain tumour imaging using APT-CEST and ${ }^{18}$ F-FET PET.

Here, we present a methodology to obtain volumetric APT-CEST and ${ }^{18}$ F-FET PET to study brain tumours using simultaneous MR-PET. We compare CEST imaging with the more clinically established ${ }^{18}$ F-FET PET to explore similarities between both imaging modalities. 


\section{Materials and Methods}

\section{Measurement details}

\section{Subjects}

Eight patients with suspected cerebral glioma were included in this feasibility study (four females, four males; median age: 56 years; age range: 20 - 69 years). All subjects presented increased ${ }^{18} \mathrm{~F}$-FET uptake in the tumour, defined as ${ }^{18} \mathrm{~F}$-FET uptake of at least 1.6 times the uptake of healthy brain tissue of the same subject [30]. Seven subjects presented a single ${ }^{18} \mathrm{~F}-\mathrm{FET}$ positive region and one subject presented two ${ }^{18} \mathrm{~F}$-FET positive regions. Detailed information on the patient group is given in Table 1 . The university ethics committee and the relevant federal authorities approved the study. All subjects provided prior written, informed consent for their participation in the study.

\section{Instrumentation}

Measurements were performed in a hybrid $3 \mathrm{~T}$ MR-BrainPET scanner (MAGNETOM Trio, Siemens Healthineers, Erlangen, Germany) equipped with a dedicated BrainPET with a spatial resolution of approximately $3 \mathrm{~mm}$ full-width at half maximum (FWHM) in the centre [31]. The BrainPET is inserted in a MAGNETOM Trio $3 \mathrm{~T}$ MR machine. A dedicated MR head coil was used, which is composed of an outer birdcage coil for transmission and an inner eight-channel receive array coil for signal reception.

\section{MR Acquisitions}

CEST measurements were performed before MR contrast agent injection in the clinical protocol. The clinical protocol includes a $\mathrm{T}_{1}$-weighted magnetization-prepared rapid gradient echo (MPRAGE) sequence and a $\mathrm{T}_{2}$-weighted fluid-attenuated inversion recovery sequence (FLAIR). A contrast-enhanced MPRAGE sequence (CE-MPRAGE) was conducted after intravenous injection of the contrast agent gadoteric acid (Dotarem, Guerbet, France).

CEST acquisition was based on the steady-state approach proposed by Ref. [32]. In our implementation, a segmented 3D EPI readout with three shots per partition was used [33]. In order to suppress the fat signal, a rectangular excitation pulse with a duration of $2.4 \mathrm{~ms}$ was used as proposed by Ref. [34]. The TR interval, consisting of a100 ms Gaussian RF pulse, crusher gradients and a partial EPI readout (echo train length $=26 \mathrm{~ms}$ ), amounted to $\mathrm{TR}=136 \mathrm{~ms}$ and an average $\mathrm{B}_{1}$ power of $1 \mu \mathrm{T}$ 
[35]. Further sequence parameters were: flip angle $=11$ degrees; TE/readout duration $=12.5 / 26 \mathrm{~ms}$; matrix size $=80 \times 80 \times 48$; voxel size $=3.0 \times 3.0 \times 3.0 \mathrm{~mm}^{3}$; sagittal slice orientation (readout superior-inferior); slice partial Fourier $=6 / 8$; phase partial Fourier $=7 / 8$ and slice oversampling of 25\%. The Z-spectrum was unevenly sampled at 69 different frequencies ranging from 14 to $-14 \mathrm{ppm}$ $( \pm 14.0, \pm 13.5, \pm 13.0, \pm 12.5, \pm 12.0, \pm 11.5, \pm 11.0, \pm 10.5, \pm 10.0, \pm 9.5, \pm 9.0, \pm 8.5, \pm 8.0, \pm 7.5, \pm 7.0, \pm 5.0$, $\pm 4.0, \pm 3.8, \pm 3.7, \pm 3.6, \pm 3.5, \pm 3.4, \pm 3.3, \pm 3.2, \pm 3.1, \pm 3.0, \pm 2.9, \pm 2.8, \pm 2.7, \pm 2.0, \pm 1.0, \pm 0.5, \pm 0.2, \pm 0.1$, 0.0) and additionally at $300 \mathrm{ppm}$ for data normalisation. Each image was acquired in 18 seconds (135 TRs; k-space centre acquired with the $\left.45^{\text {th }} \mathrm{TR}\right)$. Seamless acquisition of all images amounted to total measurement time of 21 minutes.

In addition, a 3D multi-echo gradient echo (GRE) sequence was also acquired in order to compute $\mathrm{B}_{0}$ maps. Measurement details were as follows: $\mathrm{FA}=7^{\circ} ; \mathrm{TR} / \mathrm{TE} \mathrm{E}_{1}=50 / 2.32 \mathrm{~ms} ; \Delta \mathrm{TE}=2.85$ ms; 12 echoes; monopolar readout; matrix size $=128 \times 104 \times 72$; voxel size $=1.7 \times 1.7 \times 2.0 \mathrm{~mm}^{3}$.

\section{PET Acquisition}

${ }^{18} \mathrm{~F}$-FET was produced via nucleophilic ${ }^{18} \mathrm{~F}$-fluorination with a radiochemical purity of greater than $98 \%$, a specific radioactivity greater than $200 \mathrm{GBq} / \mu \mathrm{mol}$ and a radiochemical yield of about 60 $\%$, as described previously [36]. According to the German guidelines for brain tumour imaging using labelled amino acid analogue, all patients fasted for at least $4 \mathrm{~h}$ before the PET acquisition [37]. Dynamic PET scans from 0 to 50 min post-injection were performed after intravenous injection of approximately $250 \mathrm{MBq}$ of ${ }^{18} \mathrm{~F}$-FET. Attenuation correction was performed by a template-based approach using MRI [38]. PET data were reconstructed using an OP-OSEM3D algorithm with 4 subsets and 32 iterations where normalization and attenuation, scatter, dead time and random corrections were included [39]. The reconstructed dynamic data set consisted of 16 time frames $(5 \times 1$ $\min ; 5 \times 3 \mathrm{~min} ; 6 \times 5 \mathrm{~min}$ ), image matrix of $256 \times 256 \times 153$ and voxels of $1.25 \times 1.25 \times 1.25 \mathrm{~mm}^{3}$.

\section{Data Processing}

All data processing and analyses were performed using in-house developed MATLAB code (The MathWorks, Inc., Natick, Massachusetts, United States), Python code (Python Software Foundation, version 2.7, available at http://www.python.org) and the software PMOD (Version 3.7, PMOD Technologies Ltd., Switzerland). Data co-registration was performed using the FLIRT tool 
from FSL [40], [41] using as reference the APT-CEST image at $3.5 \mathrm{ppm}$ [42].

\section{Field maps}

Field maps for $\mathrm{B}_{0}$ correction of the CEST data were calculated from the multi-echo GRE data. Images were masked based on the magnitude data and field maps were unwrapped using the algorithm from Ref. [43].

\section{CEST Data Processing}

$\mathrm{B}_{0}$ field maps were used to correct the Z-spectrum for field shifts using a smoothing spline interpolation on a voxel-by-voxel basis. After correction, the data from the offsets between 14 and 7 ppm were fitted to the Henkelman's two-pool magnetisation transfer (MT) model as suggested for the extrapolated semi-solid magnetisation transfer reference (EMR) method [43]-[46].

In order to calculate the CEST-weighted images two approaches were considered: magnetisation transfer ratio asymmetry (MTR asym) and differences to the EMR (named using \#). The $\mathrm{MTR}_{\text {asym }}$ at 3.3 to $3.7 \mathrm{ppm}$ was used, while EMR for the APT\# and NOE\# were calculated at 3.3 to $3.7 \mathrm{ppm}$ and at -3.7 to $-3.3 \mathrm{ppm}$, respectively.

\section{Data Analysis}

A volume-of-interest (VOI) analysis was performed to compare the different CEST metrics with ${ }^{18} \mathrm{~F}$ FET PET data. Three VOIs were considered to evaluate the tumour region. One PET-based tumour VOI (FET-VOI) was defined as follows: a spherical background VOI was positioned in the hemisphere contralateral to the lesion in healthy brain tissue (volume of background VOI, $14.1 \mathrm{ml} ; 7220$ voxels, 30 $\mathrm{mm}$ diameter). The tumour VOI was defined in the summed ${ }^{18} \mathrm{~F}$-FET PET image from 20 to $40 \mathrm{~min}$ post-injection by a 3D auto-contouring process using a tumour-to-brain ratio (TBR) of 1.6 or more. This metric is widely used in ${ }^{18} \mathrm{~F}$-FET PET and the cut-off value is based on a biopsy controlled study of cerebral gliomas [7]-[9], [30], [47]. Two MR-based VOIs were manually defined: FLAIR-VOI, based on the image hyperintensities; CE-MPRAGE-VOI, based on contrast-enhanced regions (Fig. 1). Statistical analysis of the TBR of the CEST metrics was performed against ${ }^{18} \mathrm{~F}$-FET data using a non-parametric Wilcoxon test using SPSS (IBM Corp. Released 2013. IBM SPSS Statistics, Version 22.0. Armonk, NY: IBM Corp). P-values of 0.05 or less were considered statistically significant. In 
addition, the hot spots in the tumour area were localized in ${ }^{18} \mathrm{~F}$-FET PET and APT\# and their distance calculated. 


\section{Results}

Figure 2 presents Z-spectra for a pixel in the tumour and for a pixel in normal appearing white matter. The signal at $3.5 \mathrm{ppm}$ is increased in the tumour voxel compared to the healthy tissue pixel. This is also visible in the difference to its corresponding EMR curve. In contrast, no peak was found in the downfield area of the Z-spectrum in healthy appearing brain tissue. In this figure it can also be seen that the EMR follows the conventional macromolecular pool for wide frequencies offsets in the range of 14 to $7 \mathrm{ppm}$.

Figures 1, 3 and 4 show the clinical and parametric images obtained for two subjects. Figure 1 shows a case with a tumour where the contrast-enhanced region did not correspond with the metabolically active area in PET. The contrast-enhanced regions in the $\mathrm{T}_{1}$-weighted MRI also did not

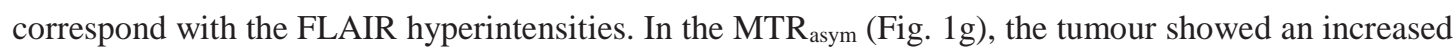
value compared to healthy appearing brain parenchyma. In the downfield APT\# images, an increased intensity was observed in the metabolically active tumour area as defined by ${ }^{18}$ F-FET PET while the NOE\# showed no differences between the tumour area and healthy appearing brain tissue. In Fig. 3, a case of a tumour with a necrotic area is presented. Here, the clinical data showed a contrast enhancement around the necrotic area, and a hyperintense signal in the FLAIR image. In the PET data the metabolically active area surrounds the necrotic region. For the CEST data, increased MTR ${ }_{\text {asym }}$ values were observed in the necrotic region, while in the EMR parametric images (NOE\# and APT\#) the necrotic region presented values near zero, suggesting a reduced effect of direct water and MT contributions. In Fig. 4, a case of a tumour in the frontal lobe is presented. Here, the clinical data showed a contrast enhancement in the tumour region, and a hyperintense signal in the FLAIR image. In the PET data the metabolically active area has a larger extension when compared to the standard

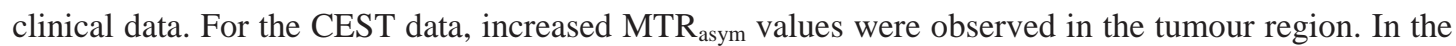
EMR parametric images, NOE\# presented no signal changes while APT\# presented an increased signal in the tumour region with a different pattern than ${ }^{18} \mathrm{~F}$-FET. This example also illustrates the advantage of using 3D CEST where the full tumour extent could be evaluated.

Table 2 presents the VOI analysis for the various metrics. The different CEST contrasts provided different information when compared with ${ }^{18}$ F-FET PET ( $\mathrm{p}<0.05$, Wilcoxon test). TBR varied across the different VOI definitions. Additionally, the location of the maxima of APT\# and ${ }^{18} \mathrm{~F}-\mathrm{FET}$ PET differ (Tab. 3). 


\section{Discussion}

The aim of this study was to compare different CEST metrics with amino acid PET in cerebral gliomas using the clinically established PET tracer, ${ }^{18} \mathrm{~F}$-FET, to examine the similarities / differences between the two methods. Each modality has already shown its diagnostic potential, for example, for tumour grading or discrimination of radiation necrosis against tumour recurrence [9], [47], [48]. While ${ }^{18}$ F-FET PET achieves a moderate accuracy of $77 \%$ for tumour grading [49], APT has shown more promising data with an area under curve in ROC analysis of $81-88 \%$ [22]. To the best of our knowledge, no study in the literature has ever compared ${ }^{18} \mathrm{~F}$-FET PET and APT. Thus, for the first time this study investigates the relation between ${ }^{18} \mathrm{~F}$-FET PET and CEST related metrics in brain tumours using simultaneous MR-PET at 3T and three-dimensional imaging.

In principle, ${ }^{18}$ F-FET PET and APT are based on different biochemical and physiological mechanisms. ${ }^{18}$ F-FET PET provides information about the expression of large amino acid transporter systems (L-type amino acid transporters 1 and 2 (LAT-1 and LAT-2)) that are more strongly expressed in brain tumours than in healthy tissue [50]. Consequently, an increased ${ }^{18} \mathrm{~F}$-FET signal is not necessarily related to increased protein synthesis or increased protein content but to an increased expression of LAT transporters. In contrast, the APT signal is related to the peptides and mobile proteins and not to the total protein content [51], as well as local modification of $\mathrm{pH}$ [52]. Our results present no relation between APT-CEST and ${ }^{18}$ F-FET PET in the tumour region (Tab. 2), suggesting that the expression of LAT transporters and transfer of peptides and mobile proteins/change of $\mathrm{pH}$ is not strictly coupled. In addition, hot-spot locations in ${ }^{18}$ F-FET and APT differ (Tab. 3), which suggests that both modalities could be used to further understand the increasingly important issue of tumour heterogeneity.

Several CEST metrics $\left(\mathrm{MTR}_{\text {asym }}, \mathrm{NOE \#} \mathrm{and} \mathrm{APT \# )} \mathrm{were} \mathrm{calculated} \mathrm{in} \mathrm{this} \mathrm{work} \mathrm{using} \mathrm{an}\right.$ irradiation power of $1 \mu \mathrm{T}$ and compared with ${ }^{18} \mathrm{~F}$-FET PET. In the literature, most studies performed at $3 \mathrm{~T}$ use an effective power of $2 \mu \mathrm{T}$ or higher [16]-[18], [22], [23], [48], [53]. The value of $2 \mu \mathrm{T}$ is chosen in order to obtain a null contrast in the normal appearing matter using $\mathrm{MTR}_{\text {asym }}$ [53], once the contribution of NOE and APT signals cancel out in healthy tissue. However, at higher powers the influence of the macromolecular pool also increases [54], resulting in APT-weighted images $\left(\mathrm{MTR}_{\text {asym }}\right)$ including MT contributions. Thus, in this work an irradiation power of $1 \mu \mathrm{T}$ was used in order to obtain nearly the maximum APT contrast and reduce the influence of the macromolecular pool that 
was further corrected using the EMR model. Moreover, this methodology proved to be stable regarding the applied power and allowed NOE\# contrast to be additionally obtained [46]. The origin of this signal is still under discussion [24]-[26] and it has also been suggested that it could be linked to protein folding and structure [56]. Moreover, studies investigating NOE effects are often performed at higher field strengths (>3 T) [24]-[26], which also influences the contrast.

In addition to various CEST metrics, different radiotracers are also available to measure amino-acid transport such as ${ }^{18}$ F-FDOPA. This radiotracer was used non-simultaneously with twodimensional CEST in Refs [28], [29]. In these studies, the authors explored the CEST signal from amines at $3 \mathrm{ppm}$ as a measure of $\mathrm{pH}$ using a saturation power of $6 \mu \mathrm{T}$. They found elevated $\mathrm{MTR}_{\mathrm{asym}}$ signal in regions with elevated uptake of ${ }^{18}$ F-FDOPA but it was not quantified.

Tissue $\mathrm{pH}$ and $\mathrm{T}_{1}$ are a potential confounding factors in the present work, as it influences the CEST contrast [16], [24], [52], [57], [58]. Regarding pH, an increased CEST signal can be associated to an alkalinisation of the tissue or an increase protein concentration that could not be disentangled in this work. However, imaging $\mathrm{pH}$ exclusively is beyond the scope of the presented work that focused on evaluating different CEST metrics in the metabolically active tumour area defined by ${ }^{18}$ F-FET PET. Regarding $\mathrm{T}_{1}$, this is also a factor that can also influence CEST contrast [16], [24]. In the tumour region, the increasing of $\mathrm{T}_{1}$ occurs usually in simultaneous with an increase of water content that may reduce the influence of this factor in CEST signal [16]. Further studies are required to understand the relationship between CEST, ${ }^{18} \mathrm{~F}-\mathrm{FET}$, pH, tumour extent, and hot-spot locations.

Defining tumour extent is an important issue for patient assessment and treatment planning. With conventional MRI, this is usually based on the contrast-enhanced regions of $\mathrm{T}_{1}$ weighted images [59]-[61]. Contrast-enhancement in MRI, however, indicates areas with a disrupted blood-brain barrier (BBB) that are, on the one hand, not specific for tumour tissue and, on the other hand, may not detect the full extent of the tumour. In contrast, ${ }^{18} \mathrm{~F}$-FET uptake is independent of the BBB integrity [62] and allows a more specific delineation of tumour extent than contrast-enhanced MRI [30]. Thus, the results cannot be compared directly and often show discrepancies. For example, in the case of low-grade gliomas where the BBB is mostly intact, often no contrast enhancement is observed, while there is still an increased uptake of ${ }^{18} \mathrm{~F}$-FET [30]. Thus, the present study also considers the ${ }^{18} \mathrm{~F}$-FET positive regions to define the metabolically tumours region. This approach is supported by the biopsycontrolled study of Ref. [30]. In the CEST literature, PET data is usually unavailable for comparison 
and consequently different studies present different strategies to delineate the tumour region that may or may not be accurate. In Ref. [18] ROIs were placed over the CE-MPRAGE or in FLAIR data when no contrast enhancement was found. In Ref. [21], [23] circular ROIs were only placed in the MTR asym with higher intensities in order to avoid the effect of averaging over larger ROIs. However, those tumour delineations do not necessarily reflect the solid and metabolic active area of the tumours and VOI values can change across different VOIs (Tab. 2). By using ${ }^{18}$ F-FET PET, the solid and metabolically active tumour tissue can be defined with high reliability; this presents an enormous advantage of the present study compared to previous studies.

Three-dimensional acquisition is required in clinical applications in order to obtain whole tumour imaging. In CEST studies, two-dimensional imaging is often used to avoid long acquisition times [24], [26], [63]. In this study a steady-state approach based on segmented 3D EPI was used [32] to obtain fast full brain coverage. It was possible to acquire a single image in 18 seconds, without the use of parallel imaging, due to hardware limitation (reduced number of receive channels). In our approach, we sampled the Z-spectrum with 70 points in $21 \mathrm{~min}$, which is still rather long but this could easily be reduced to about 10 mins by employing parallel imaging. Nevertheless, if a simplified sampling scheme with less sampling offsets is considered, as suggested in [21], the measurement time could be reduced to 6 min.

The CEST imaging technique benefits from higher static magnetic fields $(>3 \mathrm{~T})$ due to the increased spectral dispersion and increased signal-to-noise ratio. For that reason, the majority of the studies are performed at 7 T [20], [24], [32], [64]. However, no human MR-PET machine is currently available at $7 \mathrm{~T}$.

The obtained results need to be confirmed in a larger and diversified population of patients. Nevertheless, the presented methodology can be combined with high field methods [65], pointing towards human MR-PET experiments up to $9.4 \mathrm{~T}$ to allow further insights into tumour metabolism [66].

\section{Conclusions}

For the first time CEST imaging was compared with amino acid PET using ${ }^{18} \mathrm{~F}-\mathrm{FET}$ in a simultaneous MR-PET measurement. Based on the well-founded assumption that high ${ }^{18} \mathrm{~F}$-FET uptake reflects metabolically active tumour our data suggests that APT CEST provides substantially different 
information. 


\section{Acknowledgements}

We thank Lutz Tellmann, Silke Frensh, Suzanne Schaden and Kornelia Frey for assistance with the MR-PET measurements. We also thank the PET and MR groups for fruitful discussions.

\section{Funding}

This study received support from the COST Action TD1007 (COST-STSM-ECOST-STSMTD1007-010515-057663). NJS is funded in part by the Helmholtz Alliance ICEMED - Imaging and Curing Environmental Metabolic Diseases, through the Initiative and Network Fund of the Helmholtz Association. Further, NJS is supported in part by TRIMAGE, an EU FP7 project (grant agreement $\left.n^{\circ} 602621\right)$.

\section{Compliance with ethical standards}

\section{Conflict of interest}

The authors declare that they have no conflict of interest.

\section{Ethical approval}

All procedures performed in studies involving human participants were in accordance with the ethical standards of the institutional and/or national research committee and with the 1964 Helsinki declaration and its later amendments or comparable ethical standards.

\section{Informed consent}

Informed written consent was obtained from all individual participants included in the study. 


\section{References}

1. Wen PY, Macdonald DR, Reardon DA, et al. Updated response assessment criteria for highgrade gliomas: Response assessment in neuro-oncology working group. J Clin Oncol. 2010;28(11):1963-1972.

2. Heiss W-D. Clinical impact of amino acid PET in gliomas. J Nucl Med. 2014;55(8):1219-1220.

3. McConathy J, Yu W, Jarkas N, et al. Radiohalogenated nonnatural amino acids as PET and SPECT tumor imaging agents. Med Res Rev. 2012;32(4):868-905.

4. Kratochwil C, Combs SE, Leotta $\mathrm{K}$, et al. Intra-individual comparison of ${ }^{18} \mathrm{~F}-\mathrm{FET}$ and ${ }^{18} \mathrm{~F}$ DOPA in PET imaging of recurrent brain tumors. Neuro Oncol. 2014;16(3):434-440.

5. Dunet V, Rossier C, Buck A, et al. Performance of 18F-fluoro-ethyl-tyrosine (18F-FET) PET for the differential diagnosis of primary brain tumor: a systematic review and Metaanalysis. $J$ Nucl Med. 2012;53(2):207-214.

6. Heinzel A, Müller D, Langen K-J, et al. The use of O-(2-18F-fluoroethyl)-L-tyrosine PET for treatment management of bevacizumab and irinotecan in patients with recurrent high-grade glioma: a cost-effectiveness analysis. J Nucl Med. 2013;54(8):1217-1222.

7. Galldiks N, Rapp M, Stoffels G, et al. Response assessment of bevacizumab in patients with recurrent malignant glioma using [18F]Fluoroethyl-L-tyrosine PET in comparison to MRI. Eur J Nucl Med Mol Imaging. 2013;40(1):22-33.

8. Galldiks N, Stoffels G, Ruge MI, et al. Role of O-(2-18F-fluoroethyl)-L-tyrosine PET as a diagnostic tool for detection of malignant progression in patients with low-grade glioma. $J$ Nucl Med. 2013;54(12):2046-2054.

9. Ceccon G, Lohmann P, Stoffels G, et al. Dynamic O-(2-18F-fluoroethyl)-L-tyrosine positron emission tomography differentiates brain metastasis recurrence from radiation injury after radiotherapy. Neuro Oncol. 2017; 19(2):281-288.

10. Wolff S, Balaban R. NMR imaging of labile proton exchange. J Magn Reson. 1990;169:164169.

11. Ward KM, Aletrasa H, Balaban RS. A new class of contrast agents for MRI based on proton chemical exchange dependent saturation transfer (CEST). J Magn Reson. 2000;143(1):79-87.

12. Ward $\mathrm{K}$, Balaban $\mathrm{R}$. Determination of $\mathrm{pH}$ using water protons and chemical exchange dependent saturation transfer (CEST). Magn Reson Med. 2000;802:799-802.

13. Zijl P Van, Jones C, Ren J, et al. MRI detection of glycogen in vivo by using chemical exchange saturation transfer imaging (glycoCEST). Proc Natl Acad Sci USA. 2007; 104(11):4359-4364.

14. Cai K, Haris M, Singh A, et al. Magnetic resonance imaging of glutamate. Nat Med. 2012;18(2):302-306.

15. Walker-Samuel S, Ramasawmy R, Torrealdea F, et al. In vivo imaging of glucose uptake and metabolism in tumors. Nat Med. 2013;19(8):1067-1072.

16. Zhou J, Payen J, Wilson DA, et al. Using the amide proton signals of intracellular proteins and peptides to detect pH effects in MRI. Nat Med. 2003;9:1085-1090.

17. Jones CK, Schlosser MJ, van Zijl PCM, et al. Amide proton transfer imaging of human brain tumors at 3T. Magn Reson Med. 2006;56(3):585-592.

18. Zhou J, Lal B, Wilson D, et al. Amide proton transfer (APT) contrast for imaging of brain tumors. Magn Reson Med. 2003;50(6):1120-1126.

19. Tietze A, Blicher J, Mikkelsen IK, et al. Assessment of ischemic penumbra in patients with hyperacute stroke using amide proton transfer (APT) chemical exchange saturation transfer (CEST) MRI. NMR Biomed. 2014;27(2):163-174.

20. Heo H-Y, Jones CK, Hua J, et al. Whole-brain amide proton transfer (APT) and nuclear overhauser enhancement (NOE) imaging in glioma patients using low-power steady-state pulsed chemical exchange saturation transfer (CEST) imaging at 7T. J Magn Reson Imaging. 2016;44(1):41-50. 
21. Sakata A, Okada T, Yamamoto A, et al. Grading glial tumors with amide proton transfer MR imaging: different analytical approaches. J Neurooncol. 2015;122(2):339-348.

22. Zhou J, Tryggestad E, Wen Z, et al. Differentiation between glioma and radiation necrosis using molecular magnetic resonance imaging of endogenous proteins and peptides. Nat Med. 2011;17(1):130-134

23. Togao O, Yoshiura T, Keupp J, et al. Amide proton transfer imaging of adult diffuse gliomas: correlation with histopathological grades. Neuro Oncol. 2014;16(3):441-448.

24. Zaiss M, Windschuh J, Goerke S, et al. Downfield-NOE-suppressed amide-CEST-MRI at 7 Tesla provides a unique contrast in human glioblastoma. Magn Reson Med. 2017;77(1):196208.

25. Jones CK, Huang A, Xu J, et al. Nuclear Overhauser enhancement (NOE) imaging in the human brain at 7T. Neuroimage. 2013;77:114-124.

26. Paech D, Zaiss M, Meissner J-E, et al. Nuclear overhauser enhancement mediated chemical exchange saturation transfer imaging at 7 Tesla in glioblastoma patients. PLoS One. 2014;9(8):e104181.

27. Schlemmer H, Pichler B, Schmand M. Simultaneous MR/PET imaging of the human brain: feasibility study. Radiology. 2008;248(3).

28. Harris RJ, Cloughesy TF, Liau LM, et al. pH-weighted molecular imaging of gliomas using amine chemical exchange saturation transfer MRI. Neuro Oncol. 2015;17(11):1514-1524.

29. Harris RJ, Cloughesy TF, Liau LM, et al. Simulation, phantom validation, and clinical evaluation of fast $\mathrm{pH}$-weighted molecular imaging using amine chemical exchange saturation transfer echo planar imaging (CEST-EPI) in glioma at 3 T. NMR Biomed. 2016;29(11):15631576.

30. Pauleit D, Floeth F, Hamacher K, et al. O-(2-[18F]fluoroethyl)-L-tyrosine PET combined with MRI improves the diagnostic assessment of cerebral gliomas. Brain. 2005;128(3):678-687.

31. Herzog H, Langen K-J, Weirich C, et al. High resolution BrainPET combined with simultaneous MRI. Nuklearmedizin. 2011;50(2):74-82.

32. Jones C, Polders D, Hua J. In Vivo 3D whole-brain pulsed steady state chemical exchange saturation transfer at 7T. Magn Reson Med. 2012;67(6):1579-1589.

33. Stirnberg R, Pflugfelder D, Stöcker T, Shah NJ. High-Resolution 3D-fMRI at 9.4 Tesla with Intrinsically Minimised Geometric Distortions, in Proc. Intl. Soc. Mag. Reson. Med. 2013;p. 2372 .

34. Stirnberg R, Brenner D, Stöcker T, Shah NJ. Rapid fat suppression for three-dimensional echo planar imaging with minimized specific absorption rate. Magn Reson Med. 2016;76(5):15171523.

35. Zu Z, Li K, Janve V, et al. Optimizing pulsed-chemical exchange saturation transfer imaging sequences. Magn Reson Med. 2011;66(4):1100-1108.

36. Hamacher K, Coenen HH. Efficient routine production of the 18 F-labelled amino acid O- ( 2[ 18 F ] fluoroethyl ) - 1 -tyrosine. 2002;57:853-856.

37. Langen K-J, Bartenstein P, Boecker H, et al. [German guidelines for brain tumour imaging by PET and SPECT using labelled amino acids]. Nuklearmedizin. 2011;50(4):167-173.

38. Kops E, Hautzel H, Herzog H, et al. Comparison of Template-Based Versus CT-Based Attenuation Correction for Hybrid MR/PET Scanners. IEEE Trans Nucl Sci. 2015;62(5):21152121.

39. Weirich C, Scheins J, Lohmann P, et al. Quantitative PET imaging with the 3T MR-BrainPET. Nucl Instruments Methods Phys Res Sect A Accel Spectrometers, Detect Assoc Equip. 2013;702:26-28.

40. Jenkinson M, Smith S. A global optimisation method for robust affine registration of brain images. Med Image Anal. 2001;5:143-156.

41. Jenkinson M, Bannister P, Brady M, Smith S. Improved Optimization for the Robust and Accurate Linear Registration and Motion Correction of Brain Images. Neuroimage. 2002;17(2):825-841. 
42. Zhang Y, Heo H-Y, Lee D-H, et al. Selecting the reference image for registration of CEST series. J Magn Reson Imaging. 2016;43(3):756-761.

43. Abdul-Rahman HS, Gdeisat M, Burton DR, et al. Fast and robust three-dimensional best path phase unwrapping algorithm. Appl Opt. 2007;46(26):6623.

44. Henkelman R, Huang X, Xiang QS, et al. Quantitative interpretation of magnetization transfer. Magn Reson Med. 1993;29(6):759-66.

45. Morrison C, Henkelman RM. A model for magnetization transfer in tissues. Magn Reson Med. 1995; 33(4):475-82.

46. Heo H-Y, Zhang Y, Jiang S, et al. Quantitative assessment of amide proton transfer (APT) and nuclear overhauser enhancement (NOE) imaging with extrapolated semisolid magnetization transfer reference (EMR) signals: II. Comparison of three EMR models and application to human brain glioma at 3T. Magn Reson Med. 2016;75(4):1630-1639.

47. Lohmann P, Herzog H, Rota Kops E, et al. Dual-time-point O-(2-[(18)F]fluoroethyl)-Ltyrosine PET for grading of cerebral gliomas. Eur Radiol. 2015;25(10):3017-3024.

48. Sakata A, Fushimi Y, Okada T, et al. Diagnostic performance between contrast enhancement, proton MR spectroscopy, and amide proton transfer imaging in patients with brain tumors. $J$ Magn Reson Imaging. 2017; 46(3):732-739

49. Albert NL, Winkelmann I, Suchorska B, et al. Early static 18F-FET-PET scans have a higher accuracy for glioma grading than the standard 20-40 min scans. Eur J Nucl Med Mol Imaging. 2016;43(6):1105-1114.

50. Langen K-J, Hamacher K, Weckesser M, et al. O-(2-[18F]fluoroethyl)-L-tyrosine: uptake mechanisms and clinical applications. Nucl Med Biol. 2006;33(3):287-294.

51. Yan K, Fu Z, Yang C, et al. Assessing Amide Proton Transfer (APT) MRI Contrast Origins in $9 \mathrm{~L}$ Gliosarcoma in the Rat Brain Using Proteomic Analysis. Mol Imaging Biol. 2015;17(4):479-487.

52. Sun PZ, Sorensen G. Imaging pH using the chemical exchange saturation transfer (CEST) MRI: Correction of concomitant RF irradiation effects to quantify CEST MRI for chemical exchange rate and pH. Magn Reson Med. 2008;60(2):390-397.

53. Zhao X, Wen Z, Huang F, et al. Saturation power dependence of amide proton transfer image contrasts in human brain tumors and strokes at 3 T. Magn Reson Med. 2011;66(4):1033-1041.

54. Desmond KL, Stanisz GJ. Understanding quantitative pulsed CEST in the presence of MT. Magn Reson Med. 2012;67(4):979-990.

55. Heo H-Y, Lee D-H, Zhang Y, et al. Insight into the quantitative metrics of chemical exchange saturation transfer (CEST) imaging. Magn Reson Med. 2017;77(5):1853-1865.

56. Zaiss M, Kunz P, Goerke S, et al. MR imaging of protein folding in vitro employing nuclearOverhauser-mediated saturation transfer. NMR Biomed. 2013;26(12):1815-1822.

57. Griffiths J. Are cancer cells acidic? Br J Cancer. 1991;427(3):425-427.

58. Ross B, Higgins RJ, Boggan JE, et al. 31P NMR spectroscopy of the in vivo metabolism of an intracerebral glioma in the rat. Magn Reson Med. 1988;6(4):403-417.

59. Goldman S, Levivier M, Pirotte B, et al. Regional methionine and glucose uptake in high-grade gliomas: a comparative study on PET-guided stereotactic biopsy. $J$ Nucl Med. 1997;38(9):1459-1462.

60. Herholz K, Langen K-J, Schiepers C, Mountz JM. Brain tumors. Semin Nucl Med. 2012;42(6):356-370.

61. Kracht LW, Miletic H, Busch S, et al. Delineation of Brain Tumor Extent with [ 11 C ] L Methionine Positron Emission Tomography: Local Comparison with Stereotactic Histopathology. Clin Cancer Res. 2004;10:7163-7170.

62. Stegmayr C, Bandelow U, Oliveira D, et al. Influence of blood-brain barrier permeability on O(2-(18)F-fluoroethyl)-L-tyrosine uptake in rat gliomas. Eur J Nucl Med Mol Imaging. 2017;44(3)408-416.

63. Schmitt B, Zaiss M, Zhou J, Bachert P. Optimization of pulse train presaturation for CEST 
imaging in clinical scanners. Magn Reson Med. 2011;65(6):1620-1629.

64. Dula AN, Asche EM, Landman BA, et al. Developement of Chemical Exchange Saturation Transfer (CEST) at 7T. 2012;66(3):831-838.

65. Tse DHY, da Silva NA, Poser BA, Shah NJ. B1+ inhomogeneity mitigation in CEST using parallel transmission. Magn Reson Med. 2017; doi:10.1002/mrm.26624.

66. Shah NJ. Multimodal neuroimaging in humans at $9.4 \mathrm{~T}$ : a technological breakthrough towards an advanced metabolic imaging scanner. Brain Struct Funct. 2015;220(4):1867-1884. 



\section{Legends}

Figure 1. A representative patient case (glioblastoma) with FLAIR (a), CE-MPRAGE (b), ${ }^{18} \mathrm{~F}-\mathrm{FET}$ PET (c), MTR asym (g), NOE\# (h) and APT\# (j) images. The respective VOI definitions are shown in subfigures (d-f). PET shows prominent ${ }^{18}$ F-FET uptake in the tumour area in the frontal lobe (c) while APT\# (j) exhibits minor differences compared with the normal brain. When compared with the normal brain tissue, NOE\# (i) presented reduced contrast and $\mathrm{MTR}_{\text {asym }}$ (g) presented high contrast.

Figure 2. Z-Spectrum and respective EMR fitting for a tumour pixel (a) and healthy tissue pixel (b). The difference between both curves are presented in the bottom plots from where the NOE\# and APT\# can be calculated (c-d).

Figure 3. A patient case with a tumour (glioblastoma) with a necrotic region. Clinical FLAIR (a), CEMPRAGE (b), ${ }^{18}$ F-FET PET (c) and CEST metrics $\left(\mathrm{MTR}_{\text {asym }}\right.$ (d), NOE\# (e), APT\# (f)) are also presented. The tumour shows a cystic lesion in MRI $(a, b)$ and corresponding strong signal in MTR $_{\text {asym }}$ (d), which do not correspond to the active tumour depicted in ${ }^{18}$ F-FET PET. NOE\# (e) and APT\# (f)) also presented different pattern when compared with ${ }^{18} \mathrm{~F}$-FET which could not depict the rim of the lesion.

Figure 4. A patient case with a tumour (oligoastrocytoma) in the frontal lobe. Clinical FLAIR (a), CEMPRAGE (b), ${ }^{18}$ F-FET PET (c) and CEST metrics (MTR asym (d), NOE\# (e), APT\# (f)) are also presented. ${ }^{18}$ F-FET PET (c) detects clearly the metabolically active tumour tissue in suspicious areas in CE-MPRAGE (b) and FLAIR (a). CEST metrics (d-f) present different information when compared with ${ }^{18}$ F-FET. 
Figure 1

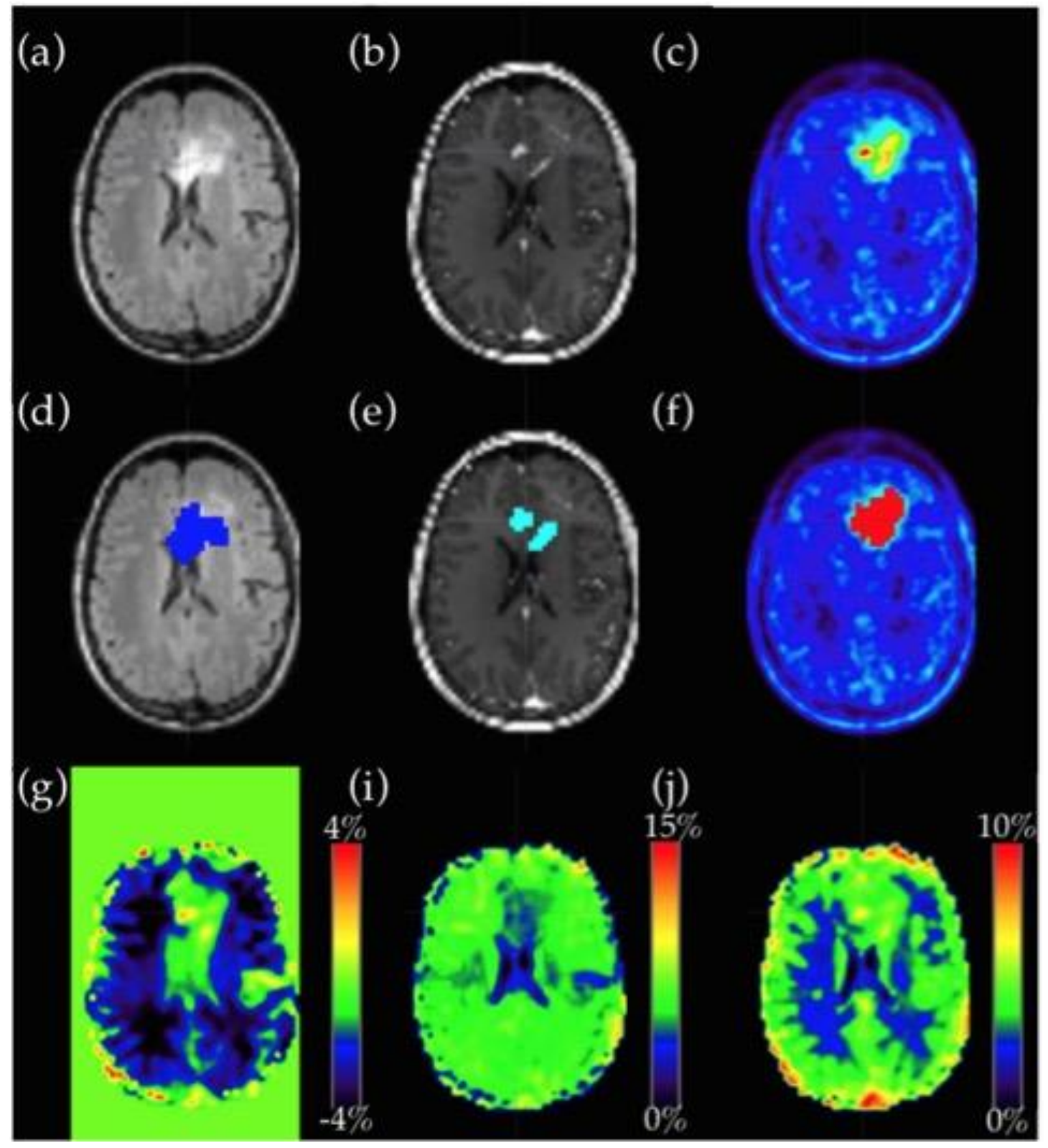


Figure 2

(a)

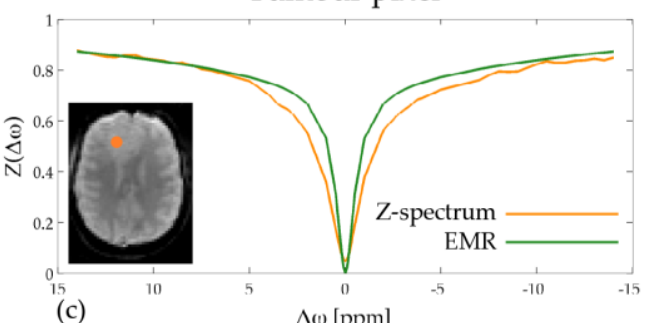

(c)

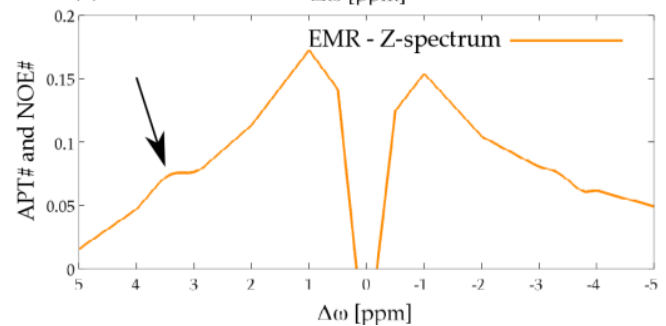

(b)

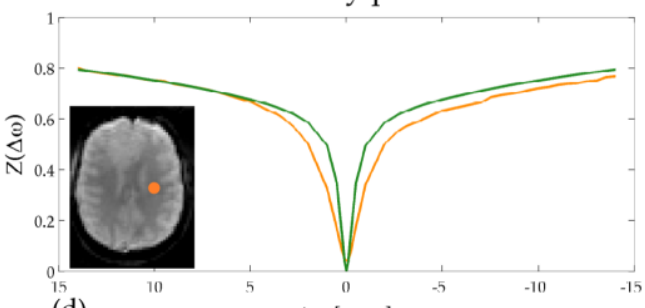

(d)

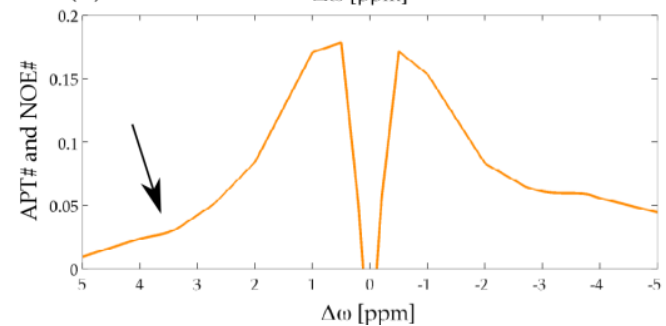


Figure 3

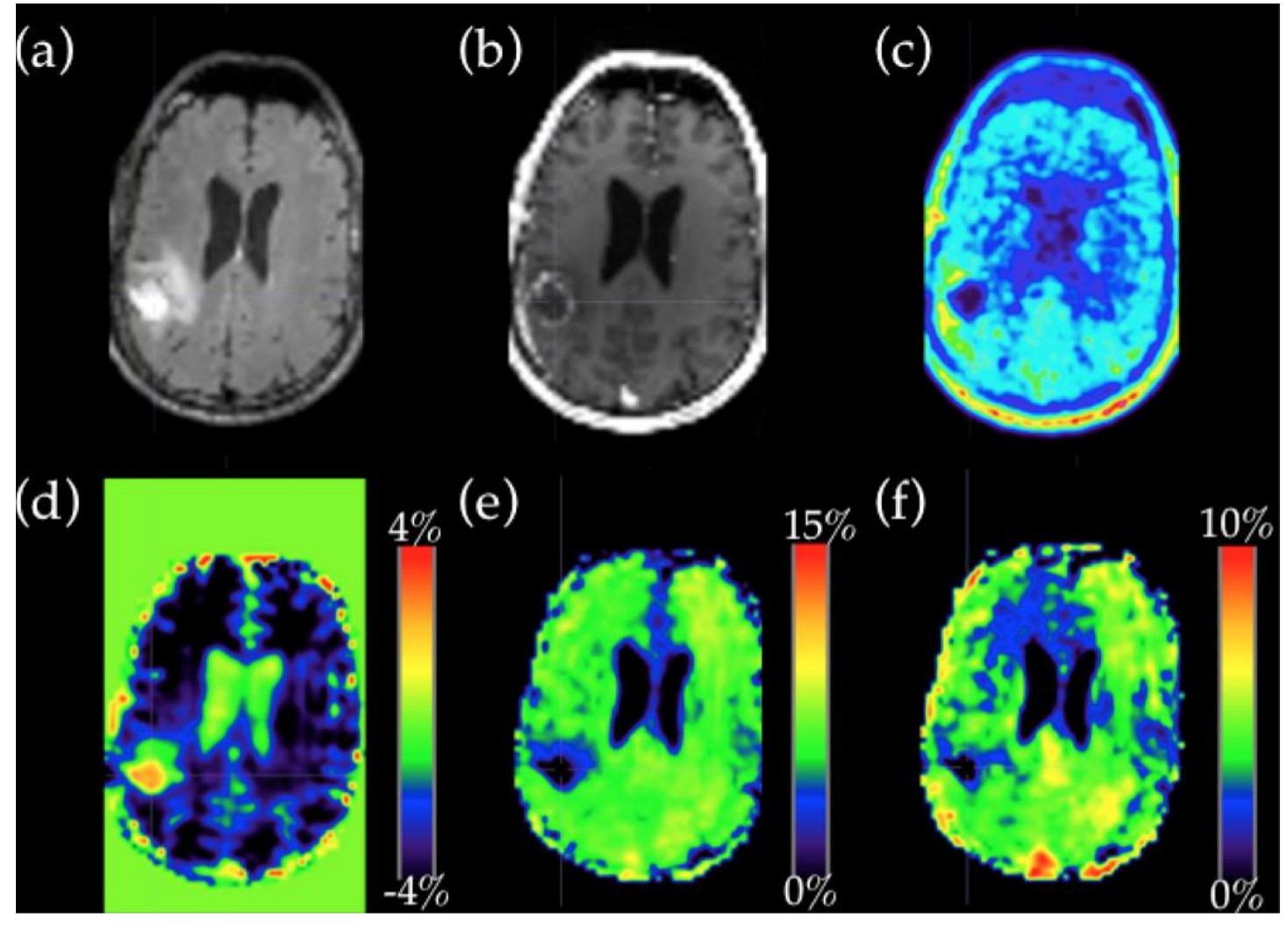


Figure 4

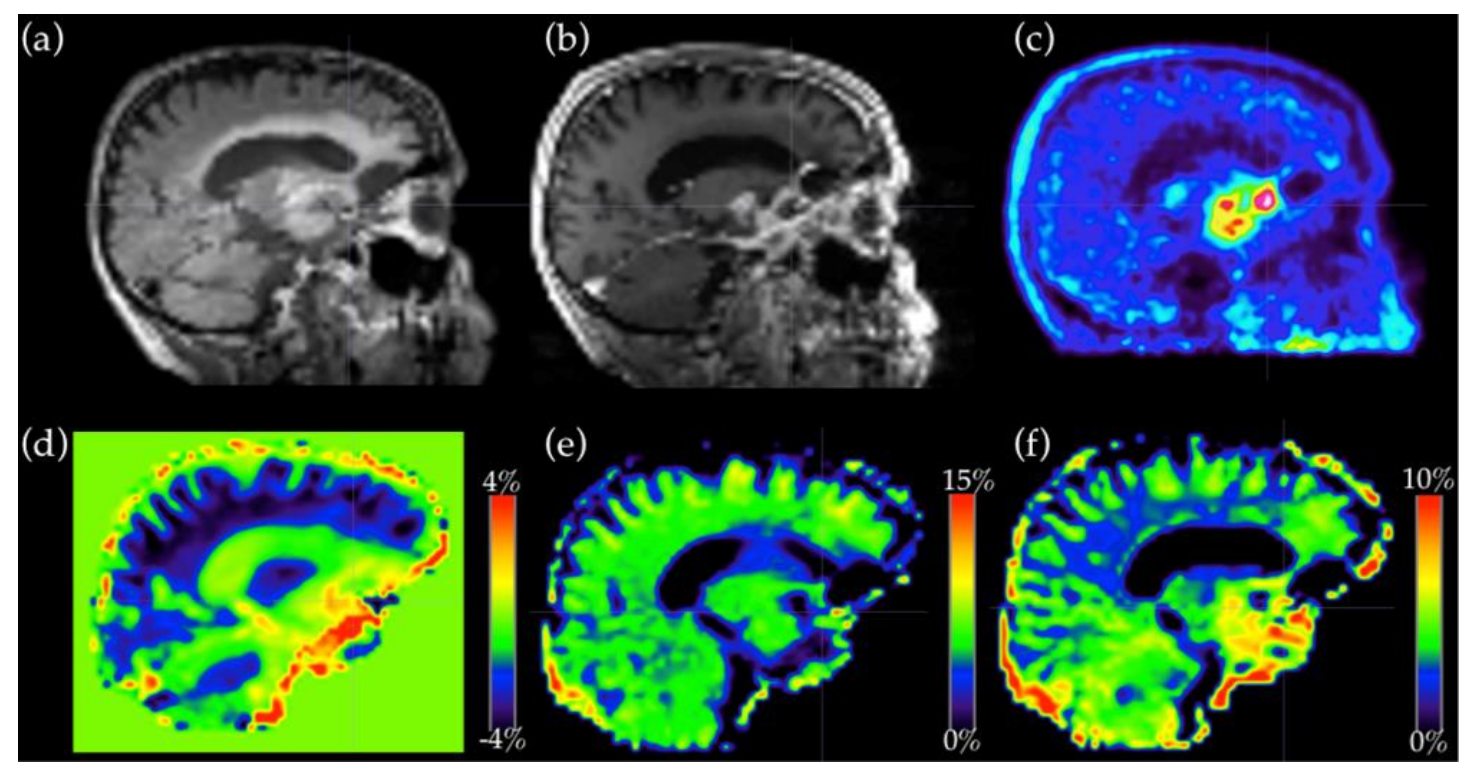




\section{Tables}

Table 1. Diagnostic and treatment data for all the patients.

\begin{tabular}{|c|c|c|}
\hline Patient ID & Diagnosis & Treatment \\
\hline 1 & $\begin{array}{c}\text { Glioblastoma/Reactive } \\
\text { changes }\end{array}$ & Radio/chemotherapy \\
\hline 2 & Glioblastoma & Radio/chemotherapy \\
(lesion 1) & Glioblastoma & Radio/chemotherapy \\
\hline 2 & Anaplastic Ependymoma & Surgery, radiotherapy \\
\hline 3 & Glioblastoma & Surgery, radio/chemotherapy \\
\hline 4 & Anaplastic Astrocytoma & None \\
\hline 5 & Glioblastomas & None \\
\hline 6 & Glioblastoma & Surgery, radio/chemotherapy \\
\hline 7 & Anaplastic & Surgery, chemotherapy \\
\hline 8 & &
\end{tabular}


Table 2. VOIs analysis of CEST data. The MR-related metrics and ${ }^{18}$ F-FET PET encode different information ( $<<0.05$, Wilkoxon test). Ranges: FLAIR-VOI (APT\#:2.93 - 4.92; NOE\#:4.04-5.66; MTR $_{\text {asym: }}$-1.89 - -0.75), CE-MPRAGE-VOI (APT\#:2.43 - 4.93; NOE\#: 3.17-5.12; MTR asym: -0.75 0.72 ) and: FET-VOI (APT\#:3.25 - 5.93; NOE\#: 3.86-6.04; MTR $\left._{\text {asym: }}-1.85-0.65\right)$

\begin{tabular}{|c|c|c|c|c|c|c|}
\cline { 2 - 7 } \multicolumn{1}{c|}{} & \multicolumn{2}{c|}{ FLAIR-VOI } & \multicolumn{2}{c|}{ CE-MPRAGE-VOI } & \multicolumn{2}{c|}{ FET-VOI } \\
\hline & Value & TBR & Value & TBR & Value & TBR \\
\hline APT\# & $3.71 \pm 0.71$ & $0.86 \pm 0.21$ & $3.89 \pm 0.91$ & $0.99 \pm 0.29$ & $4.44 \pm 0.73$ & $1.13 \pm 0.24$ \\
\hline NOE\# & $4.98 \pm 0.54$ & $1.30 \pm 1.0$ & $4.13 \pm 0.80$ & $0.71 \pm 0.15$ & $5.13 \pm 0.68$ & $0.89 \pm 0.12$ \\
\hline MTR $_{\text {asym }}$ & $-1.27 \pm 0.39$ & $0.76 \pm 0.39$ & $-0.24 \pm 0.50$ & $0.16 \pm 0.27$ & $-0.70 \pm 0.41$ & $0.41 \pm 0.41$ \\
\hline
\end{tabular}


Table 3. Distances between local hotspots defined by APT\# and ${ }^{18}$ F-FET PET.

\begin{tabular}{|c|c|}
\cline { 2 - 2 } \multicolumn{1}{c|}{} & Hotspot distances $(\mathrm{mm})$ \\
\hline Patient ID & APT\# vs. ${ }^{18}$ F-FET \\
\hline 1 & 23.4 \\
\hline $2(1)$ & 45.0 \\
\hline $2(2)$ & 27.0 \\
\hline 3 & 6.61 \\
\hline 4 & 27.17 \\
\hline 5 & 4.24 \\
\hline 6 & 4.24 \\
\hline 7 & 21.63 \\
\hline 8 & 24.19 \\
\hline
\end{tabular}

